\title{
Representing a "revolution": how the popular press has portrayed personalized medicine
}

\author{
Alessandro R Marcon, MA ${ }^{1}$, Mark Bieber, BSC $^{2}$ and Timothy Caulfield, LLM, FRSC 1,3
}

\begin{abstract}
Purpose: This study investigated the portrayal of "personalized" and "precision" medicine (PM) in North American news over the past decade. Content analysis of print and online news was conducted to determine how PM has been defined and to identify the frames used to discuss PM, including associated topics, benefits, and concerns.
\end{abstract}

Methods: A data set was built using the FACTIVA database, searching for popular North American publications with the terms "personalized (personalised) medicine" and/or "precision medicine" from 1 January 2005 to 15 March 2016. The final set of publications totaled 774

Results: PM is almost exclusively defined as related to genetics and is often part of a story related to cancer. The PM story is overwhelmingly one of highlighting (potential) benefits and optimism, especially in shorter publications, and ones where PM is not the main focus. This promotional PM discourse has remained fairly consistent over the past decade.

Conclusion: The numerous concerns associated with PM have received little attention over the past decade, especially in articles more likely to be encountered by a more general audience. This promotion of PM serves as an example of the science hyping that takes place in science reportage and may have implications for consumers, public expectations, and related health policy.

Genet Med advance online publication 4 January 2018

Key Words: content analysis; media; personalized medicine popular press; precision medicine

\section{INTRODUCTION}

Over the past decade, the hopes, benefits, and concerns as well as the use of technological and scientific advancements in the overlapping fields of genetics, medicine, science, and health care in approaches now commonly referred to as precision or personalized medicine have attracted a significant amount of media attention. The appointment of precision-medicine proponent Francis Collins to the role of director of the National Institutes of Health, the announcement of President Obama's Precision Medicine Initiative, and the emergence of numerous direct-to-consumer genetics-related health companies such as 23andMe, have all played a role in this rise of media discourse. Although the terms "personalized medicine" and "precision medicine" (PM) have become the most commonly used in this context, they still lack singular, concrete definitions. ${ }^{1,2}$ Many other terms have been used in similar contexts, including "stratified medicine," "P4 medicine," "genetic medicine," and "personalized genomics.",3 The creation and ultimate popularization of the term "PM," despite its somewhat varied use, have the fundamental roles of synthesizing under a unified title a range of genetics-related health-care practices through which information and knowledge exchanges can take place among scientists, government, industry, and the public at large. Thus, while PM, as a term, remains something of a fluid social construct, taking on diverse meanings based on the context as well as the manner in which it is used, a general, working definition of PM can be understood as "an emerging approach for disease treatment and prevention that takes into account individual variability in environment, lifestyle, and genes for each person." 4

PM is typically presented as striving toward making health care more "personalized, predictive, preventive, and participatory." 2 It has been portrayed as a means to optimize care ${ }^{5-7}$ and to empower both patients and the general public to participate more in treatment decisions as well as to take greater preventive measures. ${ }^{7}$ It has also been argued that PM will improve health-care efficiency and reduce system costs. ${ }^{8-10}$ Furthermore, it is thought not only that PM will help accelerate the development of new drugs for rare or neglected diseases ${ }^{11}$ but also that drugs previously deemed ineffective might find new uses. ${ }^{7}$ Often, when PM is promoted to the general public, it is portrayed as representing a "revolution"12 or part of a "breakthrough"13,14 in health care.

For all its potential benefits, however, PM efforts have also faced cautionary criticism. For example, commentators have questioned the clinical value of the PM approach in many contexts. ${ }^{1,15,16}$ In addition, evidence suggests that individuals are unlikely to change their behavior in response to genetic risk information, ${ }^{17,18}$ a common justification for the adoption of PM strategies. ${ }^{19}$ There is also concern that the introduction of PM might lead to increases in overdiagnosis, unnecessary testing, iatrogenic injuries, ${ }^{20}$ and health-care costs. ${ }^{21}$ In addition, the heavily promotional language often associated with PM (such as the term "revolution") has been criticized

${ }^{1}$ Health Law Institute, Faculty of Law, University of Alberta, Edmonton, Alberta, Canada; ${ }^{2}$ School of Public Health, University of Alberta, Edmonton, Alberta, Canada; ${ }^{3}$ Faculty of Law and School of Public Health, University of Alberta, Edmonton, Alberta, Canada. Correspondence: Timothy Caulfield (caulfield@ualberta.ca)

Submitted 19 June 2017; accepted 26 October 2017; advance online publication 4 January 2018. doi:10.1038/gim.2017.217 
for contributing to inaccurate public representations of science $^{22}$ and unrealistic public expectations, ${ }^{23}$ as well as facilitating a market for unproven products and services. ${ }^{24}$ Finally, some have suggested that an emphasis on PM may result in an inappropriate shift from public health initiatives to those focused on the individual. ${ }^{5,25}$ Because a large body of research has detailed issues concerning the hype ${ }^{26}$ and overly optimistic portrayals of biomedical research, ${ }^{27}$ a primary analytical focus of this study was to assess the degree to which PM has been portrayed as beneficial in light of the concerns also being raised.

With these tensions in mind, the goals of this research were to examine the portrayal of PM in popular print and online North American news media over the past decade (20052016), to determine how PM has been defined (as a process related to "genetics" or otherwise), to identify the central topics associated with PM, and to identify which-and the extent to which-PM benefits and concerns have been discussed.

\section{The role of the popular press}

Apart from the education system, mass media stands as the primary source of health information for the general public. $^{28}$ In addition, studies have shown a substantial interest in novel advancements in health care and biotechnology; one reported that almost $60 \%$ of Americans said they were "very interested" in news about medical discoveries. ${ }^{29}$ While the factors underlying the reporting and publication of health news are numerous and complex, ${ }^{30}$ the media undeniably plays a role in how the general public learns about, and makes sense of, health-related topics. For example, studies have shown how media attention on a particular topic can affect the public's utilization of health services. ${ }^{31}$ Other studies illustrate how the media plays an influential "framing" role when covering issues surrounding access to health care, at times highlighting certain aspects of debates over others ${ }^{32}$ or in some cases omitting important information concerning relevant risks and limitations. ${ }^{33}$ The concept of "framing," which has been taken up and expanded on by theorists and communication scholars, refers to the process of selecting, and in turn emphasizing, particular stories, topics, themes, facts, and actors over others. ${ }^{34}$ As a result of framing choices, certain frames resonate more with particular audiences, gaining stronger or weaker influence depending on the context. ${ }^{35}$ With regard to analyzing frames when performing content analysis, choices can be made between analyzing generic or issue-specific frames. ${ }^{36}$

\section{Data collection}

\section{MATERIALS AND METHODS}

To capture the popular representation of PM in North American news media, we used the FACTIVA database (https://www.dowjones.com/products/factiva/) to collect both print and online news stories appearing in the most popular American and Canadian news sources, including newspapers, magazines, and news-related websites. FACTIVA is a news source database owned by Dow Jones in which elaborate search inquiries can be performed and corresponding text-in this case, articles-can be downloaded. Details on the selection of news sources for this study can be found in the Supplementary Material online.

After performing a Google "news" search on the wide range of terms associated with PM (e.g., "personalized medicine," "personalized genetics," "precision medicine," "individualized medicine," "personalized treatment") and recording the number of search results for each term, we determined that "personalized medicine" (also with the spelling "personalised") and "precision medicine" were the terms used most frequently. While these two terms do not capture the totality of the PM discourse, they markedly substantiate the popularization of the concept. These two terms were searched in the FACTIVA database over the time period from 1 January 2005 to the day of collection: 15 March 2016. Because our objective was to track and analyze PM discourse over time, and because metrics such as Google Trends showed consistent use of "personalized medicine" and "precision medicine" - the latter with a sharp spike in the last 15 months - it was determined that just over a complete decade would provide an ample and sufficient data set.

A total of 1,477 articles were initially obtained. Duplicate and irrelevant articles were excluded, resulting in a final data set of 774 news articles. Articles were considered irrelevant if PM was only mentioned or briefly defined but not discussed in any detail and not connected to themes and topics represented in the story.

\section{Data analysis}

Content analysis ${ }^{37}$-a systematic and replicable process of analyzing text to make valid and objective inferences about a particular social context-was then performed on the articles to examine how PM was being framed in the media. This process involved building a coding frame through a sample analysis, which was then applied to all texts in the database. The coding results serve as an accurate portrayal of the PM communicative discourse. In this media study on PM, the frames chosen were issue-specific and were determined both inductively and deductively (an initial coding frame was modified through an analysis of a data sample), with the broad goal of mapping out which themes and topics become associated with PM. Analyzing the framing tensions between articles promoting PM and those raising concerns about PM sheds light on how the general public is being informed about these emerging technologies, and because the articles cover a time span of more than a decade, whether the story has changed or remained consistent over time. The final coding frame included (i) information (metadata) about publication date, headline, word count, author, and article source; (ii) identification of article type, e.g., news, commentary, news and commentary combined; (iii) the degree to which the article focuses on PM; (iv) definition of PM; (v) general topics associated with PM; (vi) mentions of specific health topics related to PM; (vii) mentions of specific drugs related to PM; 
(viii) benefits attributed to PM; (ix) expected timeframe for PM benefits to come into effect; (x) Concerns associated with $\mathrm{PM}$; and (xi) overall tone of the article with respect to PM. The complete coding frame can be found in the Supplementary Material, which provides the details of each coding category.

Assessment of the tone of the article was based on the presence and analysis of benefits versus concerns. An article detailing and stressing PM benefits but not PM concerns was coded as "positive." An article predominantly detailing PM benefits but also mentioning and stressing some concerns was coded as "mostly positive." The same process with the inverse values was undertaken to code articles "negative" and "mostly negative." Articles were coded "neutral" if the benefits and concerns of PM were detailed in equal measure, or if a definitive emphasis on either side could not be determined.

Because the coding aspect of content analysis can have subjective variance, we tested just over $10 \%(n=84)$ of the articles for intercoder reliability. Following a consensusreaching session in which intercoder human error was corrected and category requirements were clarified, we achieved a mean $\kappa$ score of 0.814 across all categories, demonstrating "strong" agreement based on stringent standards. ${ }^{38}$ Complete $\kappa$ scores for each category can be found in Supplementary Table S1. For category v (general topics), an agreement score ( $\kappa=0.583)$ was achieved, which illustrates only "weak" agreement. Some inconsistencies in coding for this category were found, as there were ambiguities when demarcating university research, government initiatives, and biotech industries. These data have been included in the analysis because they provide a general mapping of the topics through which PM is framed but do not weigh heavily on conclusions drawn from analysis. For category ix (timeframe of PM benefits coming into effect), we obtained weak agreement $(\kappa=0.483)$ and therefore removed all data pertaining to this category from our analysis.

\section{RESULTS}

Of the 774 articles included in the final data set, 539 (69.6\%) were from US news sources and 235 (30.3\%) were Canadian. The numbers of articles per year and the total number of articles appearing in each US and Canadian news source are shown in Supplementary Figures S1-3. With respect to article type, 391 (50.5\%) were "news," 205 (26.5\%) "news and commentary," and 178 (22.9\%) "commentary." With respect to degree of PM focus, in 338 (43.7\%) of the articles, PM was the "main focus" and in 436 (56.3\%) it was not. Regarding definitions, PM was defined as something based solely on genetics in $675(87.2 \%)$ articles, as something related to genetic procedures as well as other processes in $46(5.9 \%)$ articles, and as something not related to genetics in 53 (6.8\%) articles. Regarding general topics, PM was associated with university research in $40.1 \%$ of articles, with biotechnology in $32.9 \%$ and government initiatives in $22.9 \%$. PM was discussed in the context of specific medical procedures in $13.0 \%$ of articles and was connected to direct-to-consumer companies, products, or services in $9.9 \%$. These data are shown in Supplementary Figure S4. With respect to "other" topics associated with PM, present in only $5.4 \%$ of the articles, 18 articles focused on legal proceedings, and 5 touched on topics related to fundraising, philanthropy, and competitions. The topic of ELSI (ethical, legal, and social implications) debates was raised in four articles.

More than 120 specific health topics appear in the 774 articles, but cancer had substantially the largest presence, appearing in $441(56.9 \%)$ articles. The next most discussed health topics included diabetes, in $71(9.2 \%)$ articles; heart disease, in 56 (7.2\%) articles; cystic fibrosis, in $53(6.8 \%)$ articles; and Alzheimer disease, in 50 (6.5\%) articles. No other health topic appears in more than $6 \%$ of the total articles. For a complete list of all health topics mentioned in at least five articles, refer to Supplementary Table S2.

In $210(27.1 \%)$ articles, reference is made to specific drugs. A total of 125 different drugs are mentioned in the articles, 86 of which are mentioned only once. All drugs mentioned in at least 5 articles are listed in Supplementary Table S3.

With regard to the benefits of PM, 709 articles (91.6\%) describe eight principal PM benefits and seven "other" benefits. Of these 709 articles, 354 (49.9\%) detail only one benefit, 259 (36.5\%) detail two, and $76(10.7 \%)$ detail three. Of the 354 articles that detail only one benefit, 284 describe the efficacy of current or future PM-related treatments and 28 describe the benefit of PM-related prevention. Text examples of the efficacy of PM treatments are provided in the Supplementary Material. Sixty-five (8.4\%) articles discuss PM without detailing any benefits. The overall presence of PM benefits is displayed in Figure 1. For cases where PM was seen as beneficial in terms of creating more patient autonomy or helping to assist in the prevention of diseases, we coded the rationale stated for doing so. In these cases, increased screening and monitoring (including ease of access to screening and monitoring) were mentioned in 118 (15.2\%) articles. Positive changes regarding PM-based lifestyle changes were discussed in $67(8.7 \%)$ articles. Achieving higher levels of prevention and increasing patient autonomy

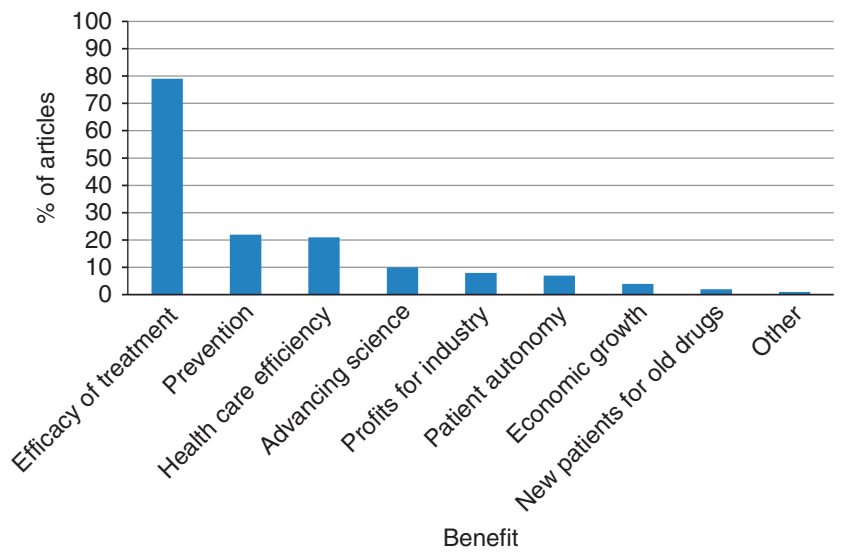

Figure 1 Mentions of personalized medicine's benefits in publications $(N=774)$. 
were equated with decreasing the burden placed on the health-care system in less than $1 \%$ of articles. PM was described as a beneficial means for "advancing science"progressing the scientific understanding of humans and environments regardless of whether a specific practical application for that knowledge could be discerned-in 77 (9.9\%) articles.

In addition to highlighting the benefits of PM, articles raised numerous PM concerns. The total number of articles raising concerns about PM, however, as displayed in Figure 2, was significantly lower than that of articles describing its benefits: 253 articles (32.7\%) raised PM concerns while 521 $(67.3 \%)$ did not.

We coded for 13 specific concerns as well as an open category labeled "other" (see Supplementary Material online for the complete list). The most common concern centered on the "limited clinical or health value of genetic information," which was expressed in $90(11.6 \%)$ articles. Economic cost concerns were mentioned in $79(10.2 \%)$ articles. The other concerns present in more than $5 \%$ of articles included "other" (or miscellaneous) in 44 (5.7\%) articles, the "exaggeration of benefits" in $43(5.6 \%)$ articles, government regulations and policies (e.g., questions about how to regulate DTC products, issues over when health care systems should use and/or fund genetic tests, etc.) in 38 (4.9\%) articles, and concerns surrounding access to and control of data, in 41 (5.3\%) articles. Concerns not present in more than $4 \%$ of articles included concerns related to genetic literacy, too much medicine (e.g., issues related to overdiagnosing, diagnostic cascade, or iatrogenic injury), genetic discrimination, patents, managing information, doctors' knowledge/expertise in interpreting genetic data, and ELSI topics pertaining to race, ethnicity, and ancestry, as well as the potential for increased inequality in health-care systems. For "other" (miscellaneous) topics, two topics made up $45.5 \%$ of the 44 : the accuracy of genetic tests (in 11 articles) and emotional issues (anxiety, false hope, and helplessness) for PM patients (in 9 articles). In terms of PM tone, the articles were overwhelmingly positive, as shown in Figure 3.

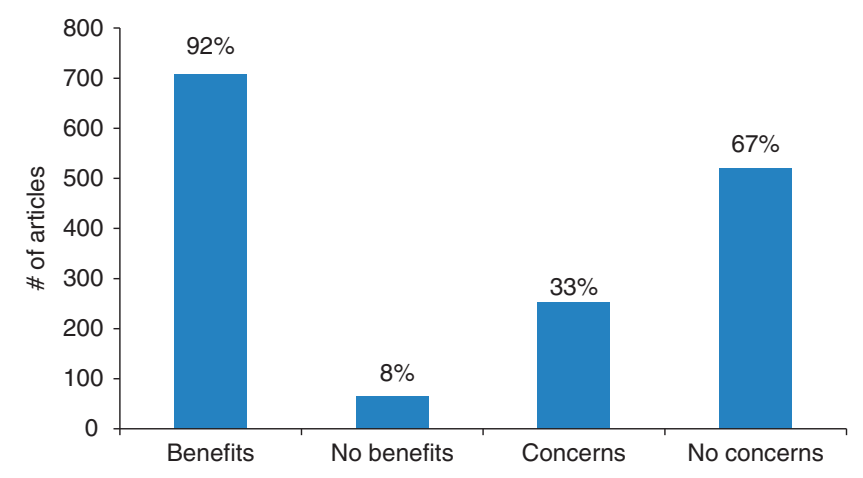

Figure 2 Mentions of personalized medicine's benefits versus concerns.

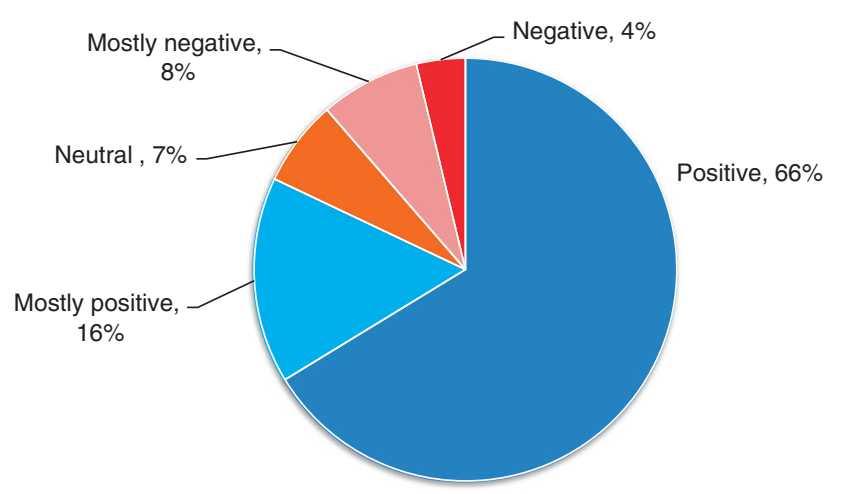

Figure 3 Overall tone of the articles with respect to personalized medicine.

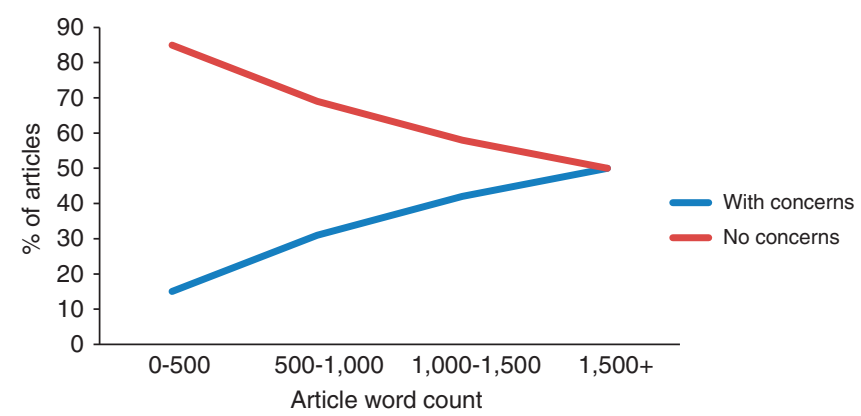

Figure 4 The percentage of articles mentioning concerns in relation to word count.

\section{PM values, the presence of concerns, and the story over time}

Concerns and PM focus

In calculating the presence of concerns when PM is the main focus versus when it is not (i.e., PM is referred to but is not the principal topic of the news story), the data reveal that more concerns are present when PM is the main focus. Of the articles in which PM is the main focus $(43.7 \%$ of all the articles), concerns are mentioned in $39.1 \%$. In contrast, of the articles in which PM is not the main focus $(56.3 \%$ of all the articles), an even smaller proportion mention concerns: $27.1 \%$ of the articles. Supplementary Figures S5 and S6 present this information.

\section{Concerns and word count}

The data show a relationship between length of article and presence of concerns. The average length of articles was approximately 1,000 words. Analysis by category-articles with fewer than 500 words, 500-1,000 words, 1,000-1,500 words, and more than 1,500 words-showed an increasing presence of concerns with increasing word count, as shown in Figure 4.

\section{PM over time}

Figure 5 displays the tone of the articles over the decade (January 2005 to March 2016). As shown, the tone-essentially 


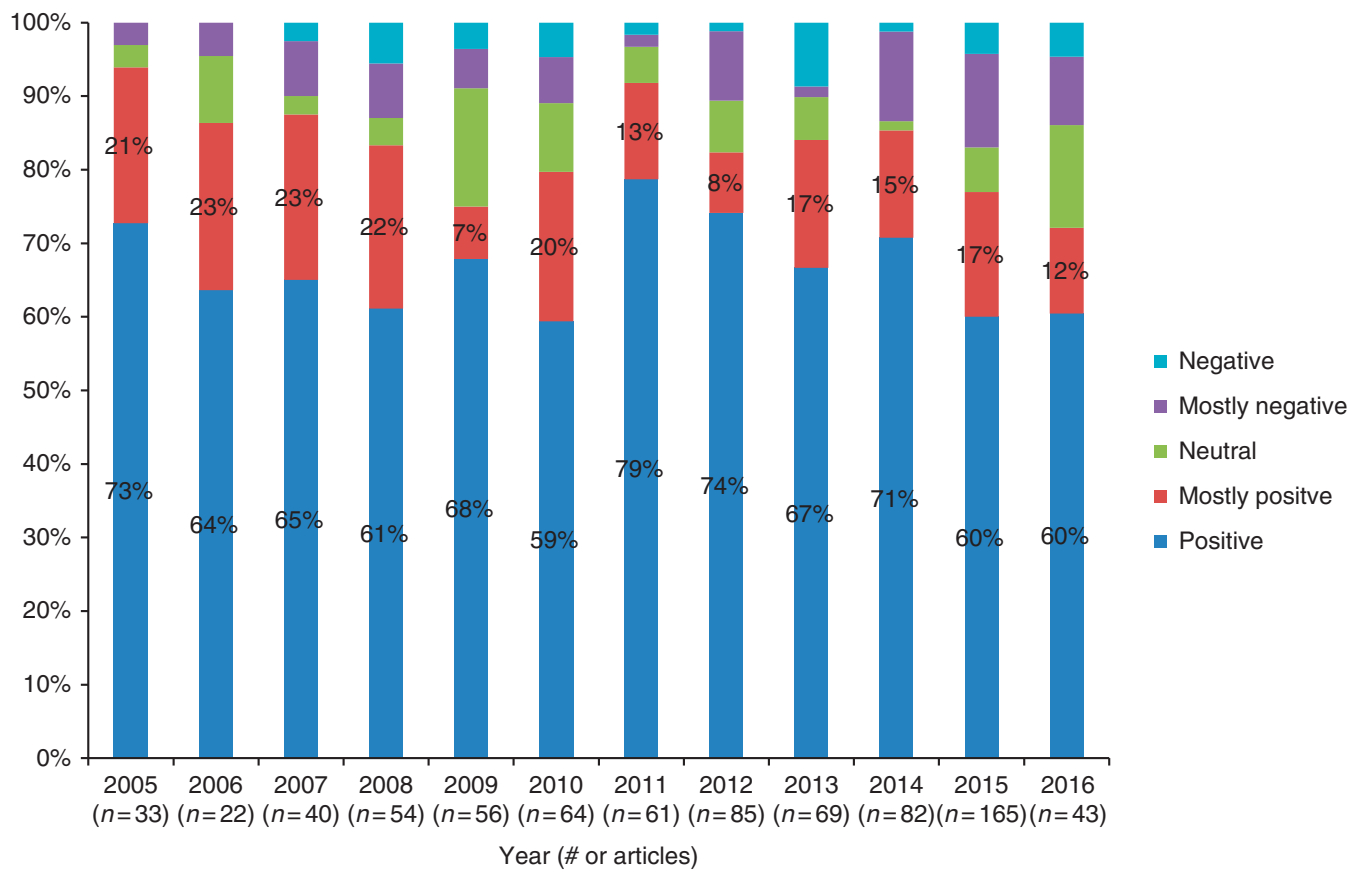

Figure 5 The overall tone of articles across the decade (up to 15 March 2016).

a portrayal of PM benefits and concerns-shows a fairly consistent pattern with no obvious directional trends.

\section{DISCUSSION}

Analyzing the PM news stories appearing over the past 10 years reveals key aspects of how PM has been framed and demonstrates that PM is largely portrayed in a promotional or positive light. Regarding PM health topics, PM is most typically presented in a story related to cancer. Other health topics, such as diabetes, heart disease, cystic fibrosis, and Alzheimer disease, receive some PM-related attention but considerably less, and PM in connection with topics such as cardiovascular disease, multiple sclerosis, autism, Crohn disease and Parkinson disease are seldom discussed in the popular press. General readers of PM-related news are therefore more likely to associate PM with cancer and are probably exposed to considerably fewer PM-related developments and issues pertaining to a wide range of health topics. Furthermore, there generally seems to be consensus in the popular press regarding the definition of PM. It is used, rightly or not, almost exclusively to define procedures, topics, or themes related to genetics. For those who believe that PM is really a broader concept-one that encompasses a variety of factors relevant to a patient, including relationships, socioeconomics, and lifestyle-this finding will undoubtedly be frustrating. However, it also speaks to how successful those invested in branding the concept of PM have been, especially since our research has observed a fairly consistent framing over the study period. In the popular sphere, PM is primarily about genetics. This raises interesting questions, worth further investigation, about whether this popular culture emphasis may facilitate an inappropriate de-emphasis of important, nongenetic, factors relevant to population health.

Another major theme is optimism. PM is almost always framed as positive or mostly positive (in $82 \%$ of the articles). Only a small percentage of the articles present PM in a negative light. Furthermore, nearly all articles (approximately 92\%) detail specific PM benefits, such as the effectiveness of current or prospective PM treatments. In contrast, the exaggeration of PM benefits, which is commonly raised by critics concerned about the lack of data surrounding $\mathrm{PM},{ }^{15}$ is mentioned in only $6 \%$ of articles. This conclusion aligns with past research that has mapped the phenomenon of science hype, ${ }^{39}$ showing genetic research to be portrayed in a predominantly positive manner and as part of a near-future "revolution."14 Future research should (continue to) explore whether the positivity surrounding the portrayal of PM is translating into an increased demand by the public for genetics-related testing. This would be especially worthwhile seeing as this study highlights increased screening and monitoring as a core PM benefit.

Another strongly promoted benefit of PM is its potential ability to encourage preventative health measures and increase patient autonomy. Specifically, several articles present PM as a way to assist individuals in adopting beneficial lifestyle changes, including screening behavior, diet, and exercise. Given that existing research suggests that the provision of genetic-risk information has little impact on behavior change, ${ }^{16,17}$ these media representations seem out of step with the existing research. Indeed, the inaccurate representations of the empowerment message stand as a good example of how the media portrayals of PM may mislead both the public and policy makers about the value of 
some PM technologies. Given that previous research has highlighted the potential impact of media representations on utilization patterns, policies, and public expectations, ${ }^{31,33}$ these results may be cause for concern. In addition, these media portrayals may facilitate the marketing of unproven direct-to-consumer genetic testing services $^{23}$ by making unsubstantiated marketing promises-which often include messages of empowerment-seem more plausible.

Given the dominance of the enthusiastic portrayals, it is somewhat ironic that the most common concerns found in the popular press focus on the limited value of genetic information and the problems of exaggerated claims of benefit (e.g., questioning the ability of PM to enable preventative measures). In other words, when concerns are found in the popular press, they often touch on the problems of overly enthusiastic portrayals of benefit. But critiques of this kind are only seldom presented in popular PM news stories.

Another interesting theme that emerged from our data was that longer and more PM-focused articles were, in general, more balanced (that is, mentioned both benefits and concerns). For example, articles focusing specifically on PM were more likely to highlight concerns (39\%), compared with articles mentioning PM as part of a broader news story (27\%). Broader news stories, therefore, including those pertaining to, for example, business/ finance, government initiatives, or technological developments, generally describe PM in a more optimistic or promotional tone than do articles that focus on PM specifically. This serves as an example of "implicit hype," a phenomenon that has been noted with other emerging health technologies, ${ }^{40}$ whereby the news media simply takes benefits for granted. Audiences encountering the topic of PM through news stories that are more general in scope are even more likely to be exposed to a positive portrayal of PM than audiences reading specifically about PM. This trend is also evident in analyzing article length. As shown in Figure 4, the longer an article is, the more likely it is to highlight concerns surrounding PM. As such, readers more on the periphery of PM-related news stories, encountering only shorter articles that describe PM using fewer words and less detail, are probably exposed to more favorable or optimistic representations of PM than those reading longer, more detailed analyses. Again, this plays into the hyping associated with $\mathrm{PM}^{26}$ which, as a result, has the potential to create not only unrealistic expectations ${ }^{14}$ but could lead to an erosion of trust and/or enthusiasm surrounding science more generally. ${ }^{27}$

The primary limitation of our study was in our choice of search terms. There is an abundance of terms associated with personalized or precision medicine. While terms such as "individualized medicine," "genetic medicine," and "personalized genomics" appeared in Google news searches with substantially less frequency than "personalized medicine" or "precision medicine," it is possible that including a wider range of search terms in our query would have resulted in a data set of articles highlighting different PM-related benefits, topics, and concerns. Further research could take this approach, exploring whether there are similar or dissimilar discourses between various PM-related terms.
Ultimately, this study captured the popularization and framing of what is now commonly known and referred to as personalized or precision medicine. It shows that in popular discourse, PM has been framed in a consistent manner: as a positive health-care trend associated with few concerns. Given the power of the media to shape public discourse, including patient expectations, this could have implications for the implementation of PM technologies. To date, the concerns associated with PM have received little attention. This is unfortunate. The public discourse would benefit from a more balanced and nuanced framing.

\section{SUPPLEMENTARY MATERIAL}

Supplementary material is linked to the online version of the paper at http://www.nature.com/gim

\section{ACKNOWLEDGMENTS}

This study was conducted under the PACEOMICS Project, funded by Genome Canada, Genome Alberta, the Canadian Institute for Health Research (CIHR), and Alberta Health \& Wellness. The project was also supported by Pegasus, which is funded by Génome Québec, Genome Canada, the Government of Canada, and the Ministère de l'Enseignement Supérieur, de la Recherche, de la Science et de la Technologie du Québec. Additionally, this study acknowledges valuable funding support from the Trudeau Foundation. The authors acknowledge the contributions made by Jenna Vivian, who assisted in the construction of the coding frame as well as the coding; Sarah Denholm, who conducted intercoder analysis; and Robyn Hyde-lay, who provided continual support and input throughout the research process.

\section{DISCLOSURE}

The authors declare no conflict of interest.

\section{REFERENCES}

1. Joyner MJ. Precision medicine, cardiovascular disease and hunting elephants. Prog Cardiovasc Dis 2016;58:651-660.

2. Juengst E, McGowan ML, Fishman JR, Settersten RA. From "personalized" to "precision" medicine: the ethical and social implications of rhetorical reform in genomic medicine. Hastings Cent Rep 2016;46:21-33.

3. Caulfield T. Genetics and personalized medicine-where's the revolution? Thebmjopinion 2015. http://blogs.bmj.com/bmj/2015/07/23/timothy-caulfieldgenetics-and-personalized-medicine-wheres-the-revolution/. Accessed 9 March 2017.

4. National Institutes of Health. All of Us Research Program. https://www.nih. gov/research-training/allofus-research-program. Accessed 9 March 2017.

5. Árnason V. The personal is political: ethics and personalized medicine. Ethical Perspect 2012;19:103-122.

6. Jameson JL, Longo DL. Precision medicine-personalized, problematic, and promising. Obstet Gynecol 2015;70:612-614.

7. Collins FS, Varmus H. A new initiative on precision medicine. New Engl J Med 2015;372:793-795.

8. Keogh B. Era of personalized medicine may herald end of soaring cancer costs. J Natl Cancer Inst 2012; 104: 12-17.

9. Flores M, Glusman G, Brogaard K, et al. P4 medicine: how systems medicine will transform the healthcare sector and society. Pers Med 2013; 10:565-576.

10. Hood L, Lovejoy JC, Price ND. Integrating big data and actionable health coaching to optimize wellness. BMC Med 2015;13:4.

11. Hamburg MA, Collins FS. The path to personalized medicine. New Engl J Med 2010;363:301-304.

12. Fayerman, P. Personalized cancer treatment on verge of revolutionizing care (with video). Vancouver Sun. 10 February 2015. http://www.vancouversun. com/health/Personalized+cancer+treatment+verge+revolutionizing+care+ with+video/11406879/story.html. Accessed 9 March 2017. 
13. Dennis B. Drugmakers find breakthroughs in medicine tailored to individuals' genetic makeups. Washington Post, 1 June 2014. https:// www.washingtonpost.com/national/health-science/drugmakers-findbreakthroughs-in-medicine-tailored-to-individuals-genetic-makeups/2014/06/ 01/40127d1c-e107-11e3-8dcc-d6b7fede081a_story.html?utm_term = . 67a08087af91. Accessed 9 March 2017.

14. Evans JP. (Mis)understanding science: the problem with scientific breakthroughs. Hastings Cent Rep 2016;46:11-13.

15. Budin-Ljøsne I, Harris JR. Patient and interest organizations' views on personalized medicine: a qualitative study. BMC Med Ethics 2016;17: 28.

16. Prasad V. Perspective: the precision-oncology illusion. Nature 2016;537: S63-S63.

17. Hollands GJ, French DP, Griffin SJ, et al. The impact of communicating genetic risks of disease on risk-reducing health behaviour: systematic review with meta-analysis. BMJ 2016:352:11102.

18. Marsaux CF, Celis-Morales C, Fallaize R, et al. Effects of a Web-based personalized intervention on physical activity in European adults: a randomized controlled trial. J Med Internet Res 2015;17:e231.

19. Collins FS. Has the revolution arrived?. Nature 2010;464:674.

20. Diamandis EP, Li M. The side effects of translational omics: overtesting, overdiagnosis, overtreatment. Clin Chem Lab Med 2016;54:389-396.

21. Petrilli CM, Heidemann L, Mack M, Durance $P$, Chopra V. Inpatient inherited thrombophilia testing. J Hosp Med 2016;11:801-804.

22. Sumner $P$, Vivian-Griffiths S, Boivin J, et al. The association between exaggeration in health related science news and academic press releases: retrospective observational study. BMJ 2014;349:g7015.

23. Petersen $A$, Krisjansen I. Assembling 'the bioeconomy': exploiting the power of the promissory life sciences. J Sociol 2015;51:28-46.

24. Caulfield T, Borry P, Toews M, Elger BS, Greely HT, McGuire A. Marginally scientific? Genetic testing of children and adolescents for lifestyle and health promotion. J Law Biosci 2015:Isv038.

25. Tedstone AE. Obesity treatment-are personalised approaches missing the point?. BMJ 2016; 354:i4980.

26. Caulfield T, Condit C. Science and the sources of hype. Public Health Genomics 2012:15:209-217.

27. Master Z, Resnik DB. Hype and public trust in science. Sci Eng Ethics 2013;19:321-335.

28. Garreu JM, Bird SJ. Ethical issues in communicating science. Sci Eng Ethics 2000;6:435-442.

29. Nisbet MC, Markowitz E. Americans' attitudes about science and technology: the social context for public communication. Commissioned review for the American Association for the Advancement of Science. 2016. https://www.aaas.org/sites/default/files/content_files/NisbetMarkowitz_ ScienceAttitudesReview_AAAS_Final_March10.pdf. Accessed 9 March 2017.
30. Gasher M, Hayes M, Hackett R, Gutstein D, Ross I, Dunn J. Spreading the news: social determinants of health reportage in Canadian daily newspapers. Can J Comm 2007;32:557.

31. Kamenova K, Reshef A, Caulfield T. Angelina Jolie's faulty gene: newspaper coverage of a celebrity's preventive bilateral mastectomy in Canada, the United States, and the United Kingdom. Genet Med 2014;16:522-528.

32. Abelson J, Collins PA. Media hyping and the "Herceptin access story": an analysis of Canadian and UK newspaper coverage. Healthc Policy 2009; 4:e113-e128

33. MacKenzie R, Chapman S, Barratt A, Holding S. 'The news is [not] all good': Misrepresentations and inaccuracies in Australian news media reports on prostate cancer screening. Med J Aust 2007:187:507-510.

34. Scheufele D. Framing as a theory of media effects. J Commun 1999;49: 103-122.

35. Nisbet MC, Newman TP. Framing, the media, and environmental communication. In: Hansen A, Cox R, (eds). The Routledge Handbook of Environment and Communication. Routledge: London, 2015.

36. Matthes J. What's in a frame? A content analysis of media framing studies in the world's leading communication journals, 1990-2005. J Mass Commun Q 2009;86:349-367.

37. Elo $S$, Kyngäs $H$. The qualitative content analysis process. J Adv Nurs 2008;62:107-115

38. McHugh ML. Interrater reliability: the kappa statistic. Biochem Med 2012;22:276-282

39. Caulfield T, Sipp D, Murry CE, Daley GQ, Kimmelman J. Confronting stem cell hype. Science 2016; 352:776-777

40. Rachul C, Rasko JE, Caulfield T. Implicit hype? Representations of platelet rich plasma in the news media. PloS One 2017;12:e0182496.

This work is licensed under a Creative Commons Attribution-NonCommercial-NoDerivs $\quad 4.0$ International License. The images or other third party material in this article are included in the article's Creative Commons license, unless indicated otherwise in the credit line; if the material is not included under the Creative Commons license, users will need to obtain permission from the license holder to reproduce the material. To view a copy of this license, visit http://creativecommons.org/licenses/ by-nc-nd/4.0/

(C) The Author(s) 2018 